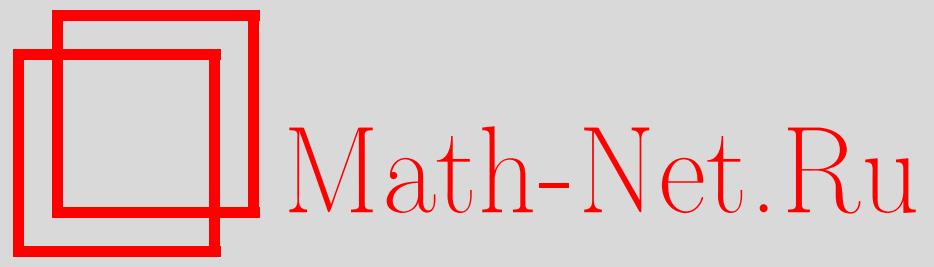

М. М. Маламуд, Л. Л. Оридорога, Теоремы полноты для систем дифференциальных уравнений, Функи. анализ и его прил., 2000, том 34, выпуск 4, 88-90

DOI: https://doi.org/10.4213/faa332

Использование Общероссийского математического портала MathNet.Ru подразумевает, что вы прочитали и согласны с пользовательским соглашением

http://www . mathnet.ru/rus/agreement

Параметры загрузки:

IP : 54.198 .187 .58

26 апреля 2023 г., 15:35:19

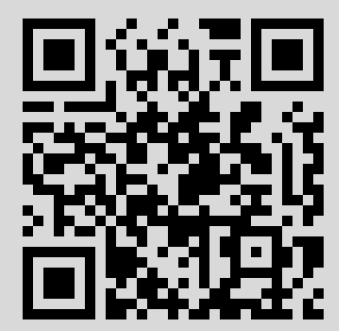


УДК 517.9

\section{Теоремы полноты для систем дифференциальных уравнений}

(С) 2000. М. М. МАЛАМУД, Л. Л. ОРИДОРОГА

1. Введение. Рассмотрим систему уравнений

$$
L y:=\frac{1}{i} B \frac{d y}{d x}+Q(x) y=\lambda y, \quad y=\operatorname{col}\left(y_{1}, \ldots, y_{n}\right)
$$

с невырожденной диагональной матрицей $B=\operatorname{diag}\left(\lambda_{1} I_{n_{1}}, \ldots, \lambda_{r} I_{n_{r}}\right) \in \mathbb{C}^{n \times n}$ и суммируемой $n \times n$-матрицей $Q$, имеющей блочное (относительно разложения $\left.\mathbb{C}^{n}=\mathbb{C}^{n_{1}} \oplus \cdots \oplus \mathbb{C}^{n_{r}}\right)$ представление $Q=\left(Q_{i j}\right)_{i, j=1}^{r}$. При $r=2, \lambda_{1}=-\lambda_{2}=1$ и $Q_{11}=Q_{22}=0$ система (1) эквивалентна системе Дирака [6]. При $r=n$ и $\lambda_{j}=\exp (2 \pi i j / n)$ к системе (1) сводится (см. [5]) дифференциальное уравнение $n$-го порядка.

Присоединим к системе (1) невырожденные граничные условия

$$
C y(0)+D y(1)=0, \quad \operatorname{Ker}\left(C C^{*}+D D^{*}\right)=\{0\},
$$

в которых $C, D \in \mathbb{C}^{n \times n}$. Обозначим, далее, через $L_{C, D}$ оператор, порожденный в $L^{p}[0,1] \otimes \mathbb{C}^{n}$ задачей (1)-(2).

В настоящем сообщении приводятся теоремы о полноте и минимальности в $L^{p}[0,1] \otimes \mathbb{C}^{n}$ системы собственных и присоединенных функций (ССПФ) оператора $L_{C, D}$.

2. Асимптотика решений. Доказательство основных результатов опирается на теорему 1 об асимптотике решений системы (1), аналогичную теореме о решениях уравнения $n$-го порядка (см. [7]).

Будем придерживаться стандартных обозначений из книги [7].

ЛЕмма 1. Пусть $\mu_{j}=\lambda_{j}^{-1} \in \mathbb{C}(1 \leqslant j \leqslant r)$. Тогда комплексная плоскость $\mathbb{C}$ может быть разбита на секторы $S_{\nu}$ так, что после некоторой перестановки чисел $\left\{\mu_{j}\right\}_{1}^{r}$ для всех $\lambda \in S_{\nu}$ справедливы неравенства $\operatorname{Re}\left(\mu_{j_{1}} \lambda\right)<\operatorname{Re}\left(\mu_{j_{2}} \lambda\right)<$ $\cdots<\operatorname{Re}\left(\mu_{j_{r}} \lambda\right)$.

Наряду с сектором $S=\left\{z \in \mathbb{C}: \varphi_{1}<\arg z<\varphi_{2}\right\}$ рассматриваются вложенные в него секторы $S_{\varepsilon}=\left\{z \in \mathbb{C}: \varphi_{1}+\varepsilon<\arg z<\varphi_{2}-\varepsilon\right\}, \varepsilon>0$.

Теорема 1. Пусть матрица $Q$ принадлежит $L^{1}[0,1] \otimes \mathbb{C}^{n \times n}$ и в блочном (относительно разложения $\mathbb{C}^{n}=\mathbb{C}^{n_{1}} \oplus \cdots \oplus \mathbb{C}^{n_{r}}$ ) представлении имеет нулевую диагональ: $Q_{j j}(x)=0, x \in[0,1](1 \leqslant j \leqslant r)$, а $S$ - один из секторов $S_{\nu}$, указанных в лемме 1. Тогда при каждом $\varepsilon>0$ и достаточно большом $R>0$ в области $S_{\varepsilon, R}:=\left\{z \in S_{\varepsilon}:|z|>R\right\}$ существует фундаментальная матрица решений $Y(x, \lambda)$ со следующими свойствами:

(a) $Y(x, \lambda)$ аналитична по $\lambda \in S_{\varepsilon, R}$;

(b) в блочном (относительно разложения $\mathbb{C}^{n}=\mathbb{C}^{n_{1}} \oplus \cdots \oplus \mathbb{C}^{n_{r}}$ ) представлении $Y(x, \lambda)=\left(Y_{k j}(x, \lambda)\right)_{k, j=1}^{r}$ она удовлетворяет равномерному по $x \in[0,1]$ 
асимптотическому соотношению

$$
Y(x, \lambda)=\left(\begin{array}{cccc}
I_{n_{1}}(x, \lambda) & O_{12}(x, \lambda) & \ldots & O_{1 r}(x, \lambda) \\
O_{21}(x, \lambda) & I_{n_{2}}(x, \lambda) & \ldots & O_{2 r}(x, \lambda) \\
\vdots & \vdots & \ddots & \vdots \\
O_{r 1}(x, \lambda) & O_{r 2}(x, \lambda) & \ldots & I_{n_{r}}(x, \lambda)
\end{array}\right) e(x, \lambda)
$$

в котором

$$
e(x, \lambda):=\operatorname{diag}\left(e^{i \mu_{1} \lambda x} I_{n_{1}}, \ldots, e^{i \mu_{r} \lambda x} I_{n_{r}}\right),
$$

$I_{n_{j}}(x, \lambda)=I_{n_{j}}+O_{j j}(x, \lambda)$, a $O_{j k}(x, \lambda):[0,1] \times S_{\varepsilon, R} \rightarrow \mathbb{C}^{n_{j} \times n_{k}}-$ матриць-фрункичии с әлементами вида о (1), аналитические по $\lambda \in S_{\varepsilon, R}$. Eсли $Q \in L^{\infty}[0,1] \otimes$ $\mathbb{C}^{n \times n}, \operatorname{mo}_{j k}(x, \lambda)=O(1 / \lambda)$.

3. Теорема о полноте. Случай $\boldsymbol{B}=\boldsymbol{B}^{*}$. Пусть матрица $B$ самосопряженная. Обозначим через $P_{ \pm}$спектральные проекторы на положительную и отрицательную части спектра матрицы $B$.

TeOpema 2. Пyсmb $Q \in L^{1}[0,1] \otimes \mathbb{C}^{n \times n}, 1 \leqslant p<\infty u$

$$
T_{1}:=P_{+} C+P_{-} D, \quad T_{2}:=P_{-} C+P_{+} D .
$$

Тогда ССПФ задачи (1) - -(2) полна и минимальна в $L^{p}[0,1] \otimes \mathbb{C}^{n}$, если

$$
\operatorname{det} T_{1} \neq 0, \quad \operatorname{det} T_{2} \neq 0 \text {. }
$$

Заметим, что с помощью калибровочного преобразования доказательство сводится к случаю матрицы $Q$, имеющей нулевую диагональ.

Остановимся кратко на некоторых частных случаях теоремы 2, а также на необходимых условиях полноты.

ПреДЛОЖЕНИЕ 1. Пусть матрицьь $C, D\left(\in \mathbb{C}^{n \times n}\right)$ удовлетворяют условию $(2)$ и неравенству $D B^{-1} D^{*}-C B^{-1} C^{*} \geqslant 0$ ( $\left.\leqslant 0\right)$. Тогда:

1) ССПФ оператора $L_{C, D}$ полна и минимальна в $L^{p}[0,1] \otimes \mathbb{C}^{n}$, если $\operatorname{det} T_{1}$ $\neq 0\left(\operatorname{det} T_{2} \neq 0\right)$.

2) Если к тому же $\operatorname{Im} Q(x) \geqslant 0(\operatorname{Im} Q(x) \leqslant 0), x \in[0,1]$, то условие $\operatorname{det} T_{1} \neq 0$ (det $\left.T_{2} \neq 0\right)$, эквивалентное паре условий $(5)$, необходимо и достаточно для полноты ССПФ оператора $L_{C, D}$ в $L^{2}[0,1] \otimes \mathbb{C}^{n}$. При этом в случае $\operatorname{det} T_{1}=0\left(\operatorname{det} T_{2}=0\right)$ дефект ССПФ в $L^{2}[0,1] \otimes \mathbb{C}^{n}$ бесконечен.

СледствИЕ 1. Пусть $C, D\left(\in \mathbb{C}^{n \times n}\right)$ удовлетворяют условию (2) и равенству $C B^{-1} C^{*}-D B^{-1} D^{*}=0$. Тогда ССПФ оператора $L_{C, D}$ полна и минимальна $в$ $L^{p}[0,1] \otimes \mathbb{C}^{n}(1 \leqslant p<\infty)$.

ЗАмЕЧАНИЯ. 1. По-видимому, условия (5) необходимы для полноты ССПФ произвольного, а не только диссипативного (см. предложение 1 ) оператора $L_{C, D}$. Это доказано пока при помощи операторов преобразования [5] для некоторых специальных типов граничных условий, а также (без операторов преобразования) в следующих случаях: (а) $B=I_{n}, Q$ произвольна; (b) $B=B^{*}$ произвольна, а $Q=0$; (с) $n=2$. Во всех перечисленных случаях дефект ССПФ бесконечен.

2. В случае $2 \times 2$-системы Дирака в [6] изучена близкая задача о полноте множества матриц-решений в пространстве матриц-функций. 
В случае простейшего оператора $L_{C, D}=-i I_{n} \otimes d / d x\left(B=I_{n}, Q=0\right)$ утверждение 2) предложения 1 довольно сложным образом доказано в [1].

3. При $p=2$ следствие 1 вытекает из известной теоремы М. В. Келдыша $[2,4]$. Действительно, в условиях следствия 1 оператор $L_{C, D}^{0}$ (вида (1)-(2) с $Q=0$ ) самосопряжен, а его резольвента имеет конечный (равный единице) порядок.

4. Теорема о полноте. Случай $\boldsymbol{B} \neq \boldsymbol{B}^{*}$. Для формулировки результата нам понадобится следующая конструкция. Пусть $A=\operatorname{diag}\left(a_{1}, \ldots, a_{n}\right)-$ диагональная матрица с (не обязательно различными) элементами $a_{k}$, не лежащими на мнимой оси, $i a_{k} \notin \mathbb{R}$. Отправляясь от произвольных матриц $C, D \in \mathbb{C}^{n \times n}$, введем вспомогательную матрицу $T_{A}(C, D)$ следующим образом: $k$-й столбец в матрице $T_{A}(C, D)$ совпадает с $k$-м столбцом матрицы $C$, если $\operatorname{Re} a_{k}>0$, и с $k$-м столбцом матрицы $D$, если $\operatorname{Re} a_{k}<0$. Ясно, что $T_{A}(C, D)=T_{-A}(D, C)$.

Теорема 3. Пусть найдутся комплексные числа $\left\{z_{j}\right\}_{1}^{3}$, такие, что

(a) нуль - внутренняя точка треугольника $\triangle_{z_{1} z_{2} z_{3}}$;

(b) $\operatorname{det} T_{z_{j} B}(C, D) \neq 0, j \in\{1,2,3\}$.

Тогда ССПФ оператора $L_{C, D}$ вида $(1)--(2)$ полна в $L^{p}[0,1] \otimes \mathbb{C}^{n}$.

Следствие 2. Если для некоторого $z \in \mathbb{C}$ матрищь $T_{z B}(C, D)$ u $T_{z B}(D, C)$ невырожденны, то ССПФ оператора $L_{C, D}$ полна в $L^{p}[0,1] \otimes \mathbb{C}^{n}$.

Это заведомо так при $C=\alpha D, \alpha \in \mathbb{C} \backslash 0$.

Остановимся кратко на связи теоремы 3 с уравнением $n$-го порядка

$$
y^{(n)}+q_{1} y^{(n-2)}+\cdots+q_{n-1} y=\lambda^{n} y .
$$

Как известно, полноту ССПФ краевой задачи с нерегулярными распадающимися краевыми условиями для уравнения (6), анонсированную М. В. Келдышем [3], впервые доказал А. А. Шкаликов [8]. В [5] показано, что уравнение (6) сводится к системе (1) с матрицей $B=\operatorname{diag}\left(\varepsilon_{1}, \ldots, \varepsilon_{n}\right)$, где $\varepsilon_{j}=\exp (2 \pi i j / n)$. При этом из теоремы 3 вытекает полнота ССПФ некоторых классов граничных задач со спектральным параметром, рационально входящим в граничные условия. Метод доказательства теоремы 3 является развитием метода, примененного в [8].

Отметим, однако, что изученная в [8] задача приводит к задаче (1)-(2) с матрицами $C$ и $D$, полиномиально зависящими от спектрального параметра. Этим задачам будет посвящена отдельная работа.

\section{ЛИТЕРАТУРА}

1. Гинзбург Ю. П. Функц. анализ и его прил., 5, вып. 3, 32-41 (1971). 2. Гохберг И. Ц., Крейн M. Г. Введение в теорию линейных несамосопряженных операторов. Наука, М., 1965. 3. Келдыи М. В. ДАН СССР, 77, №1, 11-14 (1951). 4. Келдыи М. В., Лидский В. Б. В кн.: Труды IV Всесоюзного математического съезда, т. 1, 1963, с. 101-120. 5. Маламуд М. М. Труды ММО, 60, 199-258 (1999). 6. Марченко В. А. Операторы Штурма-Лиувилля и их приложения. Наукова думка, Киев, 1977. 7. Наймарк М. А. Линейные дифференциальные операторы. Наука, М., 1968. 8. Шкаликов А. А. Функц. анализ и его прил., 10, вып. 4, 69-80 (1976). 